\title{
Transient heating effects on tungsten: ablation of Be layers and enhanced fuzz growth
}

\author{
J.H. Yu ${ }^{a^{*}}$, M.J. Baldwin ${ }^{\mathrm{a}}$, R.P. Doerner ${ }^{\mathrm{a}}$, T. Dittmar ${ }^{\mathrm{b}}$, A. Hakola ${ }^{\mathrm{c}}$, T. Höschen ${ }^{\mathrm{d}}$, J. \\ Likonen $^{\mathrm{c}}$, D. Nishijima ${ }^{\mathrm{a}}$, and H.H. Toudeshki ${ }^{\mathrm{a}}$
}

\author{
${ }^{a}$ Center for Energy Research, University of California at San Diego, La Jolla, CA 92093-0417, \\ USA \\ ${ }^{b}$ Institut für Energie- und Klimaforschung: Plasmaphysik (IEK-4), 52425 Jülich, Germany \\ ${ }^{c}$ VTT, PO Box 1000, 02044 VTT, Finland \\ ${ }^{d}$ Max-Planck-Institut für Plasmaphysik (IPP), D-85748 Garching, Germany
}

\begin{abstract}
A pulsed laser in the PISCES-B facility is used to simulate transient heating events such as ELMs and disruptions on W. The first study of enhanced nano-scale W tendril growth ("fuzz") due to cyclic fast transient heating of $\mathrm{W}$ exposed to low energy ( $\left.E_{\mathrm{He+}} \sim 30 \mathrm{eV}\right) \mathrm{He}^{+}$ions is presented. Fuzz due to transient heating is up to $\sim 10 \mathrm{x}$ thicker than the steady state fuzz thickness with no laser heating. A general thermal activation model yields higher values for the activation energy and pre-exponential factor than previously reported in steady state experiments with $E_{\mathrm{He}^{+}} \sim 60$ $\mathrm{eV}$. Transient heating of $\mathrm{W}$ exposed to D plasma with Be seeding shows that the removal threshold of Be follows simple energy considerations based on the heat of formation of Be.
\end{abstract}

PACS: 52.40.Hf, 52.55Fa, 61.46.+w, 52.38.Mf

PSI-20 keywords: Tungsten, Beryllium, Laser, Divertor, PISCES B

*Corresponding author address: 9500 Gilman Dr., MC 0417, La Jolla, California 92093, USA

*Corresponding author e-mail: j2yu@eng.ucsd.edu 


\section{Introduction}

Tungsten (W) is an attractive plasma facing material for future fusion reactors due to its high melting temperature and low sputtering yield. Transient heating of $\mathrm{W}$ by a pulsed laser during steady-state plasma exposure in PISCES-B is used to simulate heat loads expected to occur in future tokamaks due to edge localized modes (ELMs) and disruptions. We study here the effects of transient high heat flux on mixed Be/W surfaces and on pure $\mathrm{W}$ exposed to He plasma. The mixed material studies are motivated by the expected beryllium (Be) flux to the W divertor due to eroded Be from ITER's first wall. Previous work characterized the Be surface changes including Be-W alloying [1]. Here we study the ablation of Be layers on W.

Helium (He) formed from D-T reactions in future fusion reactors will impact the performance of $\mathrm{W}$ as a plasma facing material. It is well known that the surface of $\mathrm{W}$ exposed to He ions ( $\gtrsim 20 \mathrm{eV}$ ) develops nanometer-scale filament structures known as fuzz [2] when the steady-state surface temperature is between 1000-2000 K. Previous work found that high heat fluxes on pre-grown fuzz caused fuzz erosion [3] as well as fuzz to be reintegrated back into the bulk [4,5]. Here we show the first results of W fuzz growth due to fast transient heating, with heat flux factors and heating timescales similar to those expected during ELMs in ITER.

\section{Experimental Setup}

An Nd:YAG laser (wavelength of $1064 \mathrm{~nm}$ with adjustable pulse width from 1 to $10 \mathrm{~ms}$ and maximum peak power of $5 \mathrm{~kW}$ ) is used to irradiate $2.5 \mathrm{~cm}$ diameter $\mathrm{W}$ disks with laser spot diameter 0.5 to $1.2 \mathrm{~mm}$. The laser is guided by a set of turning mirrors 
over a distance of $\sim 20 \mathrm{~m}$ and a lens is used to obtain the desired spot size at the target. The reflectivity of bare $\mathrm{W}$ is measured in order to give an estimate of the absorbed laser power. The samples are exposed to D or He plasma during laser irradiation, and the laser strikes the target at an angle of 15 degrees with respect to normal.

$\mathrm{Be} / \mathrm{D}$ codeposits are created on W substrates in the PISCES-B facility [6] using a Be evaporative oven during D plasma exposure. The typical ion flux is $0.5-1.0 \times 10^{23}$ $\mathrm{m}^{-2} \mathrm{~s}^{-1}$ with plasma exposure duration ranging from 1000 to $5000 \mathrm{~s}$, Be layer thickness ranging from $\sim 100 \mathrm{~nm}$ to $3 \mu \mathrm{m}$, and sample temperature during plasma exposure of $50 \mathrm{C}$ for water-cooled samples. During Be injection, the concentration of $\mathrm{Be}^{+}$ions is 0.1 to $0.01 \%$ as determined from spectroscopic measurements of the Be II line at $313.1 \mathrm{~nm}$. Thicker Be layers up to $50 \mu \mathrm{m}$ are created in a Be magnetron sputtering device [7]. Samples were analyzed using secondary ion mass spectrometry (SIMS) and sputter x-ray photoelectron spectroscopy (XPS) to obtain depth profiles of Be, D, and W.

In experiments with $\mathrm{He}$ plasma exposure and no $\mathrm{Be}$ injection, the base temperature of the W sample is controlled by air cooling and was set to $880-1090 \mathrm{~K}$, which is close to the onset threshold of fuzz formation ( 1000 K). The sample is biased to $-50 \mathrm{~V}$, and the He ion flux is above the "saturation" regime where fuzz growth is independent of ion flux. After laser and plasma exposure the samples were moved in air for analysis with a scanning electron microscope (SEM).

\section{Results}

\subsection{Be ablation threshold}


A simple estimate of the incident energy density required to remove a layer of Be is based on the Be heat of formation, $\Delta H$. We call this quantity the Be formation energy density, given by $\varepsilon_{B e}=\Delta H \rho d / \mu$, where $\rho$ is the density of Be, $d$ is the Be layer thickness, and $\mu$ is the Be molar mass. To test this removal threshold, a series of single laser shots was fired on a target with a relatively thick Be layer of $d=50 \mu m$ during $\mathrm{D}$ plasma exposure. Figs. 1a - c show SEM images of the damaged Be layer due to three laser energy densities of $1.3,4.1$, and $11.9 \mathrm{MJm}^{-2}$, respectively. For context, data points in Fig. 1d show the various Be layers and laser energy densities that have been studied in the present work, and the solid line shows $\varepsilon_{B e}$. The data points labeled a, b, and c correspond to the SEM images. The Be layer is expected to survive when $\varepsilon_{\text {laser }}<\varepsilon_{B e}$, which is shown in Fig. 1a where the Be layer partially melts and the laser pulse does not penetrate to the underlying $\mathrm{W}$. When $\varepsilon_{\text {laser }} \approx \varepsilon_{\text {Be }}$, there is partial removal of the Be layer and evidence of additional molten Be as seen in Fig 1b. When the central laser energy density $\varepsilon_{\text {laser }}>\varepsilon_{B e}$, the Be is removed and the underlying $\mathrm{W}$ is visible as seen in Fig. 1c.

To get additional insight into Be removal from the $\mathrm{W}$ surface by the laser, the Be I signal $(\lambda=457.3 \mathrm{~nm})$ was measured with a spectrometer ( $1 \mathrm{~ms}$ temporal resolution) viewing nearly normal to the $\mathrm{W}$ target (15 degrees). Fig. 1e shows the time-integrated (20 ms) Be I signal measured during each laser shot that correspond to Figs. 1a-c. The Be I signal begins saturating when $\varepsilon_{\text {laser }}>\varepsilon_{B e}$, and this saturation is attributed to the removal of Be by the heat pulse resulting in limited availability of Be on the surface.

In contrast to the relatively thick Be coating and single laser shots, the regime of a thin Be coating with repetitive fast transients was studied post-mortem using SIMS on the 
data point marked by the arrow in Fig. 1d. Fig. 2a shows SIMS depth profiles of W, Be, and D on a sample with a $\sim 350 \mathrm{~nm}$ thick Be codeposit created in PISCES-B during D plasma exposure. The sample was hit with 7000 laser pulses with pulse width of $1 \mathrm{~ms}$ and absorbed laser energy density $\sim 0.3 \mathrm{MJ} \mathrm{m}^{-2}$. Fig. $2 \mathrm{~b}$ shows that Be and $\mathrm{D}$ were removed from the surface, as only trace amounts of Be and D are visible. During the course of laser pulsing at $2 \mathrm{~Hz}$, D fluence to the laser spot was $3.9 \times 10^{26} \mathrm{~m}^{-2}$. The laser was moved to a new location on the target with the plasma still on, and the spot received an additional D plasma fluence of $2.2 \times 10^{26} \mathrm{~m}^{-2}$ with Be seeding. The relatively small amount of Be and D in the laser irradiated spot even with additional plasma fluence suggests that implantation was affected in the heated region.

\subsection{Be-W alloying}

In addition to Be ablation, W-Be alloying has been detected using sputter XPS on a sample irradiated with a single laser shot. A shift was detected in the XPS spectra between the laser irradiated and non-irradiated areas, indicating the formation of Be-W alloy [1]. The sample had a $\sim 3 \mu \mathrm{m}$ thick Be layer with absorbed heat flux factor of $\sim 70$ $\mathrm{MJ} \mathrm{m}^{-2} \mathrm{~s}^{-1 / 2}$ (10 ms pulse width), which is well within the regime $\varepsilon_{\text {laser }}>\varepsilon_{B e}$ as shown by the data point at $\varepsilon_{\text {laser }}=7 \mathrm{MJ} \mathrm{m}^{-2}$ in Fig. $1 \mathrm{~d}$. The detection of Be-W alloying after one transient heat pulse shows that a single large ELM in ITER can compromise the material properties of the heat-affected area.

\subsection{Fuzz growth induced by transient heating}


In experiments during He plasma exposure, we report the first observations of W fuzz growth due to cyclic, fast transient heating. The $\mathrm{He}^{+}$ion flux to the sample was $\sim 1.0 \times 10^{23} \mathrm{~m}^{-2} \mathrm{~s}^{-1}$, and the base temperature of $\mathrm{W}$ was maintained close to the minimum temperature for fuzz growth. The sample was repeatedly irradiated by the laser with pulse width 1 ms and repetition frequency between 1 and $5 \mathrm{~Hz}$ in order to simulate ELMs.

The fuzz thickness depends on the peak temperature reached during the transient heating cycle, and decreases further from the center of the laser spot due to the temperature profile. Fig. 3a shows a top-view of a laser spot on a cleaved sample (for cross-sectional SEM imaging) after 2800 laser heating cycles. The labels show the locations of SEM images in Fig. 3c-h. We model the temporal and spatial dependence of the temperature in the $\mathrm{W}$ disc using the 3D code ANSYS, and for a laser heat flux matching experimental conditions, the calculated surface temperature profile at the end of a $1 \mathrm{~ms}$ pulse is shown in Fig. 3b with $0.5 \mathrm{GWm}^{-2}$ of absorbed heat intensity. The laser spatial profile is assumed to be flat and the absorption homogeneous within the laser spot. The base temperature of the sample with no laser irradiation is $1093 \mathrm{~K}$ and a thin ( 400 $\mathrm{nm}$ ) layer of fuzz develops with no laser heating during the $3600 \mathrm{~s}$ plasma exposure, as seen in Fig. 3g-h. Fuzz thickness is up to 10x larger in the center of laser irradiated spot compared to the non-irradiated area.

The fuzz length $l$ is modeled simply as a thermally activated process with $\sqrt{t}$ scaling, and the fuzz length is given by $l=2 \sqrt{\int D(t) d t}$, where $D(t)$ is a time dependent diffusivity. This approach is motivated by the $\sqrt{t}$ scaling observed in steady state fuzz experiments [8]. The fuzz length depends on time through its temperature dependence in an Arrhenius relationship, $D(t)=D_{0} \exp \left(-E_{0} / T(t)\right)$, where $D_{o}$ and $E_{o}$ are the pre- 
exponential factor and the activation energy, respectively. Fig. 4a shows the measured temperature evolution during a single laser pulse using a high speed pyrometer [9], and the modeled temperature (dashed line). Also shown is $D$ calculated using parameters for our data (black), and using Baldwin's parameters (blue) obtained from steady state experiments [8] with $\mathrm{He}$ ion energy $E_{\mathrm{He}+} \sim 60 \mathrm{eV}$. An important difference between Baldwin's data and our data is that we intentionally kept $E_{\mathrm{He+}} \sim 30 \mathrm{eV}$ to avoid laserinduced arcing [10] (although arcing did occur for the highest energy laser pulses).

The modeled temperature profile is repeated according to the number of laser pulses and duty cycle used in the experiments, illustrated in Fig. 4b, and the resulting fuzz evolution is calculated from $l$ as shown in Fig. 4c. Our data with $E_{\mathrm{He+}}$ close to the fuzz formation threshold shows that fuzz growth proceeds in a step-wise fashion at each heating pulse. However, fuzz growth using Baldwin's parameters is dominated by the base temperature between heating pulses and the growth proceeds smoothly as $\sqrt{t}$ with virtually no effect from fast transient heating.

The data points in Fig. 4d show the dependence of the fuzz thickness on the peak temperature reached during each laser pulse, and the peak temperature is based on 3D modeling of the temperature within the laser spot. Above $\sim 2000 \mathrm{~K}$ there is large scatter in the data because fuzz becomes reintegrated into the $\mathrm{W}$ bulk $[4,11,12]$. The red data points (no error bars shown) at $\mathrm{T}>2200 \mathrm{~K}$ shows reintegration completely dominates growth and negligible fuzz is detected. The large error bars in the peak temperature are from uncertainties in modeling, in addition to the changing reflectivity and $\mathrm{W}$ thermal conductivity [5] as the surface evolves. The red and black lines are the calculated fuzz length $l$ using $E_{o}=2.2 \mathrm{eV}$ and $D_{o}=8.0 \times 10^{-3} \mathrm{~cm}^{2} \mathrm{~s}^{-1}$ for two separate cycle numbers 
1000 and 2800, respectively. With no laser pulsing (shown by the two open symbols near $1100 \mathrm{~K})$, the measured fuzz length is smaller than that predicted using Baldwin's parameters, shown as the blue line with $E_{o}=0.71 \mathrm{eV}$. In addition, Baldwin's parameters predict that the fuzz length is insensitive to peak transient temperature $T_{\text {peak }}$, while our data have a clear dependence on $T_{\text {peak }}$. The $\mathrm{He}^{+}$ion energy of $E_{\mathrm{He+}} \sim 30 \mathrm{eV}$ used here is close to the ion energy threshold for fuzz formation [13], so it may not be surprising that a higher activation energy is required.

\section{Discussion and Conclusion}

Laser heating of Be-coated $\mathrm{W}$ supports the simple threshold concept for Be layer removal based on the Be heat of formation. If the arrival rate of Be at the $\mathrm{W}$ divertor is sufficiently large, a surface layer of Be could form which might protect the $\mathrm{W}$ substrate during transient events [14]. However, there are two problems if relying on Be coatings as a protective measure for $\mathrm{W}$ in ITER: 1) the Be layer thickness required to protect the underlying substrate from the expected ELM energy is larger than the expected layer thickness in ITER [15], and 2) the concept of a sacrificial Be layer is undermined by BeW alloying. A single high heat flux transient event that causes alloying can compromise the thermomechanical properties of the material compared to that for pure W. When BeW alloying occurs we have found that the material has a lower cracking threshold compared to pure $\mathrm{W}[1]$.

W fuzz formation has been observed for the first time using cyclic, fast transient heating. At sufficiently high heat pulses, fuzz growth is inhibited by reintegration in the W bulk. We find discrepancy between Baldwin's parameters and the data shown here at 
$E_{\mathrm{He+}} \sim 30 \mathrm{eV}$, which indicates 1$)$ the model needs modification to describe both steady state and transient fuzz growth, or 2) the activation energy $E_{o}$ and pre-factor $D_{o}$ depend on $\mathrm{He}^{+}$energy. In [16], fuzz grown in Alcator C-Mod over the course of 14 plasma discharges showed agreement with Baldwin’s parameters (using a different analysis than what was done here). In those experiments the timescale for the temperature evolution was much slower ( $\sim 1 \mathrm{~s}$ ), so if a different model is needed, apparently the timescale for the temperature evolution must be faster than seconds.

The results indicate that predictions for fuzz growth in the ITER divertor should not only consider steady-state heat loads, but also transient heating events such as ELMs. Although technically challenging due to arcing, in future experiments with fast transient heating $E_{\mathrm{He+}}$ will be increased to further investigate fuzz formation during transient heating.

\section{Acknowledgements}

This work was supported by US DOE grant DE-FG02-07ER54912. 


\section{References}

1. J.H. Yu et al., Phys. Scr. T159 (2014) 014036.

2. S. Takamura, et al., Plasma Fusion Res. 1 (2006) 051.

3. D. Nishijima, et al., J. Nucl. Mater. 434 (2013) 230.

4. G. De Temmerman et al., J. Nucl. Mat. 438 (2013) S78.

5. S. Kajita, et al., Nucl. Fusion 47 (2007) 1358.

6. R.P. Doerner at al., Phys. Scripta T111 (2004) 75.

7. A. Nikroo et al., Phys. Plasmas 13 (2006) 056302.

8. M.J. Baldwin and R.P. Doerner, Nucl. Fusion 48 (2008) 035001.

9. J.H. Yu et al., J. Nucl. Mater. 438 (2013) S1150.

10. S. Kajita et al., Nucl. Fusion 49 (2009) 032002.

11. M.J. Baldwin and R.P Doerner, J. Nucl. Mat. 404 (2010) 16.

12. S. Kajita et al., J. Nucl. Mater. 440 (2013) 55.

13. M.J. Baldwin et al., J. Nucl. Mater. 415 (2011) S104.

14. C.P.C. Wong, J. Nucl. Mater. 390 (2009) 1026.

15. K. Schmid, Nucl. Fusion 48 (2008) 105004.

16. G.M. Wright et al., J. Nucl. Mater. 438 (2013) S84. 


\section{Figure Captions}

Fig. 1 a)-c) SEM images of a $50 \mu \mathrm{m}$ thick Be layer on $\mathrm{W}$ after a single laser pulse with laser energy densities $\varepsilon_{\text {laser }}=1.3,4.1$, and $11.9 \mathrm{MJm}^{-2}$ with pulse widths 1,3 , and $10 \mathrm{~ms}$, respectively. d) Data points show the experimental parameters relative to the Be formation energy density $\varepsilon_{B e}$. The labels a, b, and c correspond to the SEM images. e) The Be I signal as function of the laser energy density.

Fig. 2 SIMS depth profiles of Be, D, and W with and without laser irradiation. Shaded region shows the approximate depth of the original Be/D layer.

Fig.3 a) Top view of the sample showing the laser spot, seen as the light-colored region. The sample is intentionally broken for cross-section imaging, and the dark area shows the edge of the break. b) Calculated surface temperature at the end of a heating pulse. Scale is the same as in a). The SEM images in c) - f) show top and cross-section views of the locations marked in a).

Fig. 4 a) Measured and modeled (red dashed line) surface temperature for a single heating cycle. The Arrhenius coefficient $D$ is calculated from the modeled temperature using parameters obtained from our data (black) and Baldwin's data (blue). b) Illustration of 5 heating cycles used for modeling. c) The growth of the fuzz thickness calculated 
from $l=2 \sqrt{\int D(t) d t}$ using the heat pulses shown in b) and the model parameters written in a). Data points in d) show the measured fuzz thickness after 1000 (red squares) and 2800 (black circles) heating cycles. Solid black and red lines are the calculated fuzz thicknesses using identical parameters. Blue line is the calculated fuzz thickness using Baldwin's parameters and 2800 cycles; solid line indicates the range of steady state temperatures used in Baldwin’s work [8]. 
Figure 1 (two column)
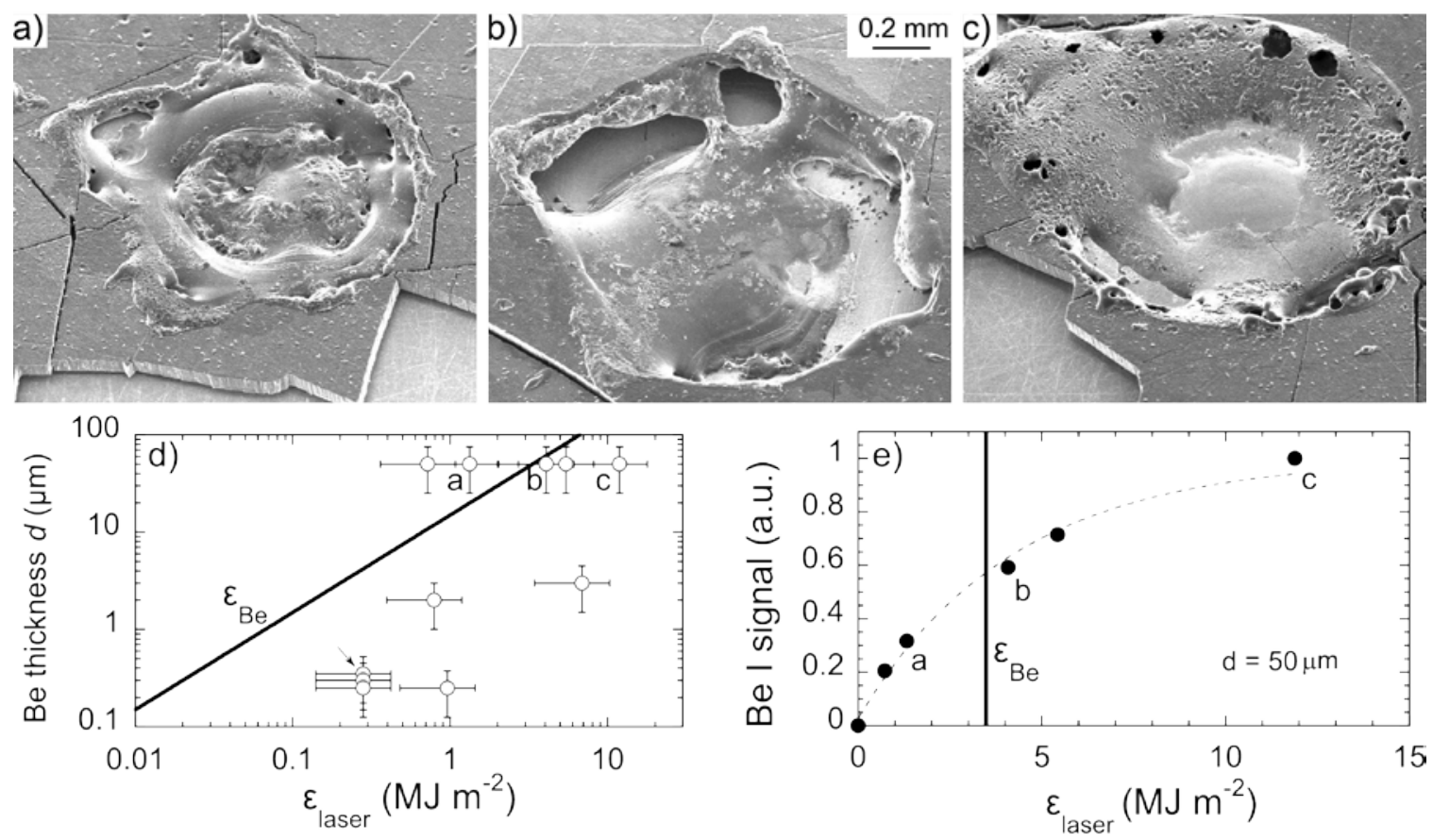
Figure 2
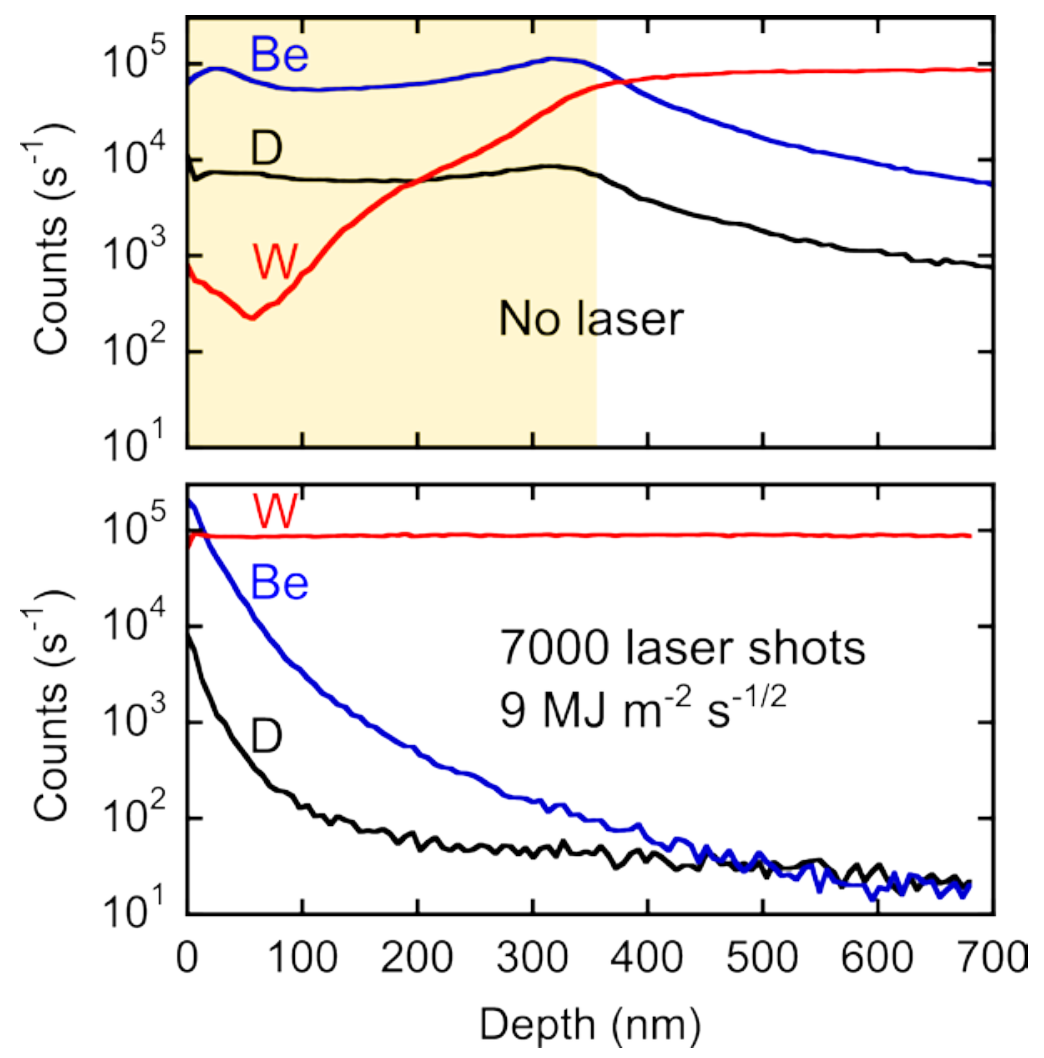
Figure 3 (two column)
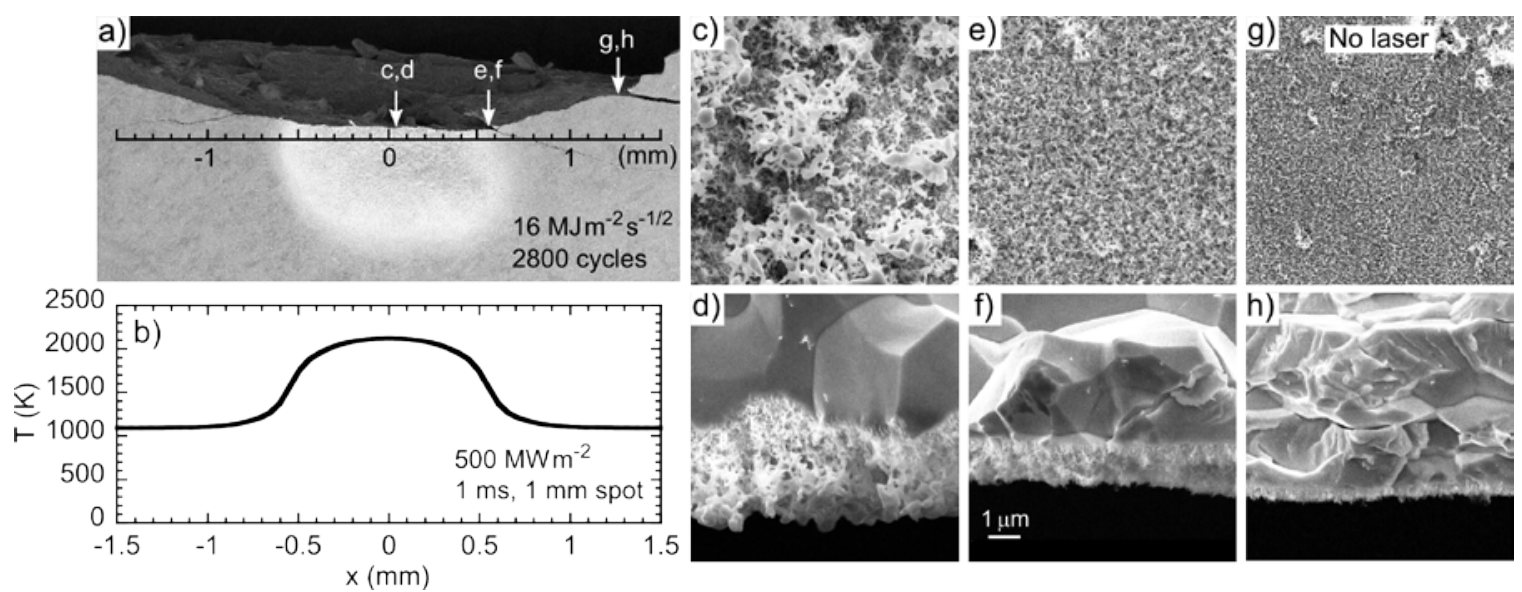
Figure 4
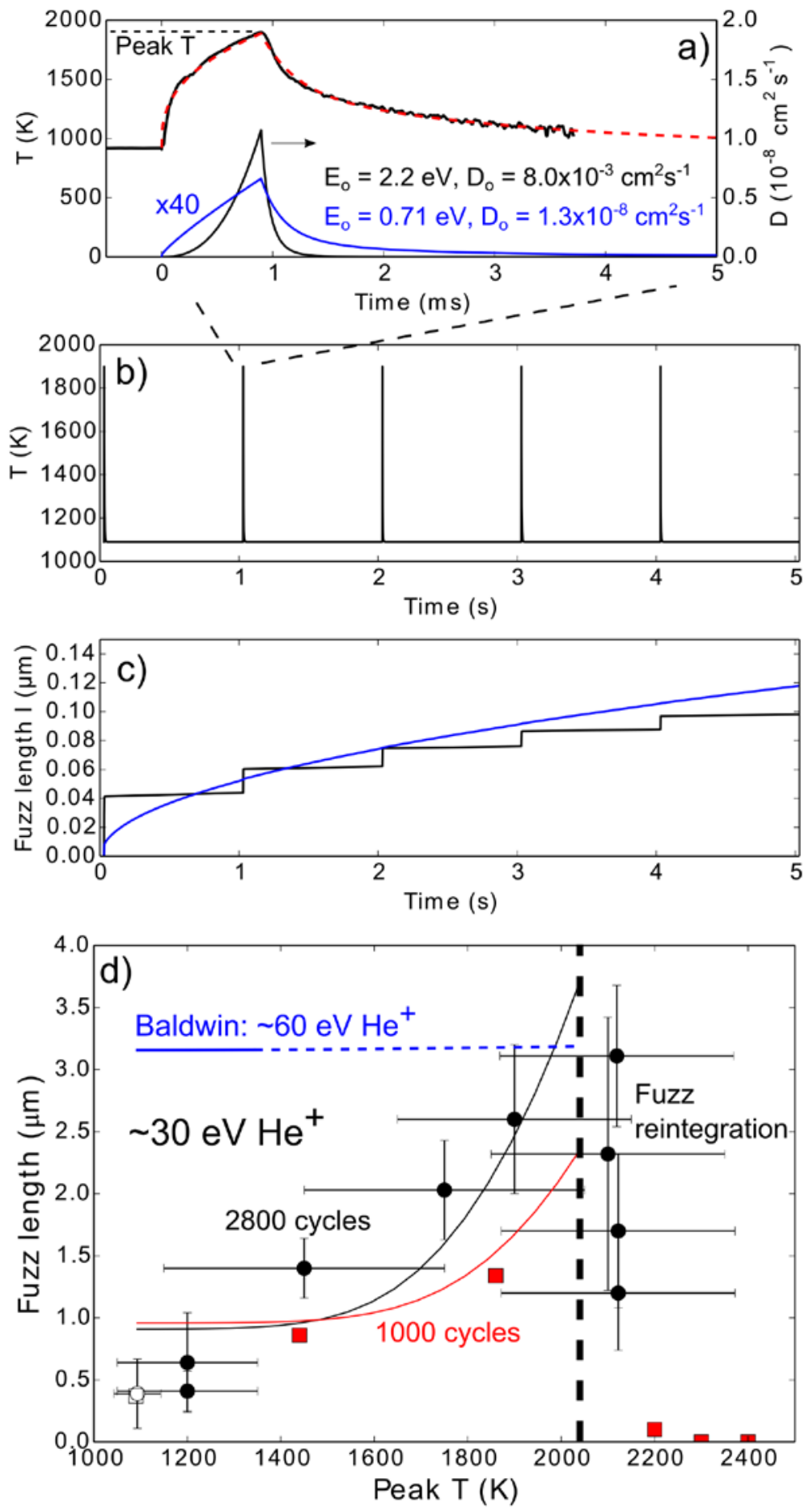